\title{
PROPOSIÇÃO DE MODELOS PARA ESTIMATIVA DA ERODIBILIDADE DE LATOSSOLOS BRASILEIROS 1
}

\author{
MARX LEANDRO NAVES SILVA ${ }^{2}$, NILTON CUR ${ }^{3}$, MOZART MARTINS FERREIRA ${ }^{3}$, \\ JOSÉ MARIA DE LIMA ${ }^{3}$ e DANIEL FURTADO FERREIRA ${ }^{4}$
}

\begin{abstract}
RESUMO - Os métodos existentes para determinação indireta da erodibilidade não têm se mostrado adequados quando aplicados aos solos tropicais, particularmente no tocante aos latossolos brasileiros. Sendo assim, objetivou-se, com este estudo, desenvolver modelos para a estimativa da erodibilidade desse grupamento de solos, a partir de variáveis morfológicas, físicas, químicas e mineralógicas do solo. Foram feitas em 19 latossolos das várias regiões geográficas brasileiras, 129 determinações (variáveis independentes), cujos resultados foram submetidos à análise de regressão linear múltipla em "backward" utilizando o programa SAS, relacionando-se com a erodibilidade obtida diretamente no campo através de parcelas de perdas de solo (variável dependente). Foi possível desenvolver cinco modelos, com graus crescentes de complexidade e precisão para a estimativa da erodibilidade dos latossolos brasileiros, englobando 37 determinações que apresentam significância.
\end{abstract}

Termos para indexação: erosão hídrica, perdas de solo.

\section{PROPOSITION OF MODELS FOR ERODIBILITY ESTIMATION OF BRAZILIAN OXISOLS}

\begin{abstract}
The existent methods for indirect determination of erodibility have not been adequate when applied to tropical soils, mainly in relation to Brazilian (Oxisols). So, this study aimed to develop models for determination of erodibility of this soils group, from morphological, physical, chemical and mineralogical variables of the soil. In this way, using 19 latosols from the various Brazilian geographical regions, 129 determinations (independent variables) were made, whose results were submitted to multiple linear regression analyses in backward using the SAS program, correlating them with the erodibility directly obtained in the field through soil losses plots (dependent variable). It was possible to develop five models, with increasing degrees of complexity and precision, for the estimation of the erodibility of Brazilian latosols, involving 37 determinations that presented significance.
\end{abstract}

Index terms: Latosols, hydrical erosion, soil losses.

\section{INTRODUÇÃO}

O solo é um recurso natural que tem um papel destacado dentro dos ecossistemas terrestres, e estando nesta posição, recaem sobre ele as mais variadas formas de degradação, como a erosão hídrica,

\footnotetext{
${ }^{1}$ Aceito para publicação em 24 de fevereiro de 1999.

Extraído da tese de Doutorado apresentada pelo primeiro autor à Universidade Federal de Lavras (UFLA) MG.

${ }^{2}$ Eng. Agr., D.Sc., Dep. de Ciência do Solo, UFLA, Caixa Postal 37, CEP 37200-000 Lavras, MG. E-mail: marx@ufla.br

${ }^{3}$ Eng. Agr., Ph.D., Dep. de Ciência do Solo, UFLA E-mail: niltcuri@ufla.br, mozartmf@ufla.br e joslima@ufla.br

${ }^{4}$ Eng. Agr., D.Sc., Dep. de Ciências Exatas, UFLA E-mail: dafurtado@ufla.br
}

que é um dos aspectos mais importantes a ser considerado com relação ao seu uso e manejo. No presente estudo, o conceito de erodibilidade expressa a resistência do solo à erosão hídrica. Entre outros fatores, é um reflexo dos atributos mineralógicos (El-Swaify \& Dangler, 1977), químicos, morfológicos e físicos do solo (Dumas, 1965; Wischmeier \& Mannering, 1969; Wischmeier et al., 1971; Lombardi Neto \& Bertoni, 1975; El-Swaify \& Dangler, 1977; Römkens et al., 1977; Young \& Mutchler, 1977; Denardin, 1990; Lima et al., 1990; Paez \& Ildefonso, 1992; Chaves, 1994; Marques et al., 1997b).

O uso dos valores de erodibilidade, quando incorporado à equação para estimar perdas de solo por erosão hídrica - USLE - (Wischmeier \& Smith, 
1978), permite a elaboração de mapas de vulnerabilidade à erosão, através do uso do Sistema de Informações Geográficas (SIG), bem como a avaliação da erosão hídrica em sistemas de manejo do solo, a composição de modelos para determinação de espaçamento de terraços, e a seleção de técnicas de conservação do solo em áreas cultivadas, visando contribuir para a sustentabilidade dos sistemas de produção agrícola.

Em termos de conservação do solo, a erodibilidade do solo constitui-se no fator de maior custo e morosidade para determinação, notadamente no Brasil, em face de extensão territorial e da diversidade edáfica (Denardin, 1990). Outro aspecto está relacionado ao método empregado para determinação da erodibilidade, pois a determinação direta, através da instalação de parcelas de perdas de solo no campo, seja sob chuva natural ou simulada, envolve altos custos, além de demandar vários anos de coleta de dados. Por esses motivos, vários autores têm desenvolvido modelos visando à estimativa da erodibilidade de maneira indireta (Dumas, 1965; Wischmeier \& Mannering, 1969; Wischmeier et al., 1971; Lombardi Neto \& Bertoni, 1975; El-Swaify \& Dangler, 1977; Römkens et al., 1977; Young \& Mutchler, 1977; Denardin, 1990; Lima et al., 1990; Paez \& Ildefonso, 1992; Chaves, 1994; Marques et al., 1997b).

A obtenção indireta da erodibilidade do solo por meio de modelos matemáticos que empregam atributos do solo tem sido usada com sucesso nos EUA (Denardin, 1990; Renard et al., 1994). Entretanto, os métodos existentes para determinação indireta da erodibilidade, desenvolvidos por Wischmeier \& Mannering (1969), Wischmeier et al. (1971) e Römkens et al. (1977), não se têm mostrado adequados quando aplicados aos solos tropicais. Essa inadequação tem sido atribuída às diferenças texturais, particularmente no tocante aos latossolos brasileiros (Freire \& Pessotti, 1974; Angulo, 1983; Henklain \& Freire, 1983; Martins Filho \& Silva, 1985; Silva et al., 1986; Denardin, 1990; Silva et al., 1994). Mesmo os métodos desenvolvidos ou adaptados para a região tropical (El-Swaify \& Dangler, 1977; Obi et al., 1989; Denardin, 1990; Lima et al., 1990) não se têm mostrado adequados (Silva et al., 1994; Marques et al., 1997a). Uma das causas dessa inadequação é a gran- de heterogeneidade de comportamento dos solos tropicais em face do processo erosivo (El-Swaify \& Dangler, 1977). Sendo assim, diversos autores têm sugerido o desenvolvimento de modelos para estimativa da erodibilidade, específicos para cada grupamento de solos (El-Swaify \& Dangler, 1977).

Objetivou-se, com este estudo, desenvolver, para latossolos brasileiros, modelos para estimativa de sua erodibilidade, a partir de atributos morfológicos, físicos, químicos e mineralógicos, facilmente determináveis em campo ou em laboratório.

\section{MATERIAL E MÉTODOS}

Para o desenvolvimento deste estudo foram utilizadas amostras da camada superficial $(0-0,20 \mathrm{~m})$ de 19 latossolos localizados em áreas experimentais nas várias regiões geográficas brasileiras, a saber: latossolo Roxo (LR) em Ijuí (RS), Chapecó (SC), Londrina (PR), Campinas (SP), e Dourados (MS); Latossolo Vermelho-Escuro (LE) em Passo Fundo (RS), Ponta Grossa (PR), Paranavaí (PR), Bela Vista do Paraíso (PR), Jaboticabal (SP), Lavras (MG), Sete Lagoas (MG), Goiânia (GO) e Planaltina (DF); Latossolo Vermelho-Amarelo (LV) em Lavras (MG), Areia (PB) e Ubajára (CE); Latossolo Amarelo (LA) em Manaus (AM) e Tomé-Açú (PA), segundo Silva (1997).

A classificação dos solos e os atributos morfológicos abordados neste trabalho basearam-se na descrição geral e morfológica dos perfis do solo (Silva, 1997), segundo recomendações de Lemos \& Santos (1996). Com as informações contidas na descrição geral, codificou-se a drenagem do perfil; com as informações morfológicas, codificou-se a cor (úmida) segundo classificação de Munsell (Munsell Color Company, 1975), a estrutura (tipo, grau e tamanho) e a consistência quanto à plasticidade (solo molhado). Para a drenagem do perfil foram usadas as seguintes classes: excessivamente drenado $=1$; fortemente drenado $=2$; acentuadamente drenado $=3$; bem drenado $=4$; moderadamente drenado $=5$; imperfeitamente drenado $=6$. A cor do solo foi descrita segundo Munsell (Munsell Color Company, 1975), e o matiz foi codificado da seguinte forma: $10 \mathrm{R}=1 ; 2,5 \mathrm{YR}=2 ; 5 \mathrm{YR}=3 ; 10 \mathrm{YR}=4 ; 2,5 \mathrm{Y}=5$. As classes de estrutura foram codificadas, quanto ao tipo, em: blocos angulares $=1$; blocos subangulares $=2$; granular $=3$; grumos $=4$. $\mathrm{O}$ grau de desenvolvimento da estrutura foi codificado da seguinte forma: grãos simples (não coerente) $=1$; fraca $=2 ;$ moderada $=3 ;$ forte $=4 ;$ maciça $($ coerente $)=5$; quanto ao tamanho: muito pequena $=1$; pequena $=2$; média $=3 ;$ grande $=4 ;$ muito grande $=5$. A consistência do solo quando molhado foi codificada como: não plástico $=1$; 
ligeiramente plástico $=2$; plástico $=3$; muito plástico $=4$. Quanto à pegajosidade, têm-se as seguintes classes: não pegajoso $=1 ;$ ligeiramente pegajoso $=2 ;$ pegajoso $=3$; muito pegajoso $=4$.

A análise granulométrica dos solos foi realizada pelo método da pipeta (Day, 1965), empregando-se $\mathrm{NaOH} 0,1 \mathrm{~mol} \mathrm{~L}^{-1}$ como dispersante químico e agitação rápida $(12.000 \mathrm{rpm})$ durante dez minutos. $\mathrm{O}$ fracionamento da terra fina em água também foi realizado pelo método da pipeta. As frações que englobam partículas ou agregados maiores que $0,1 \mathrm{~mm}$ foram separadas por peneiramento (peneira de $0,105 \mathrm{~mm}$ ), e as frações de tamanho menor, por sedimentação, segundo a lei de Stockes. A fração areia (2,0 a $0,053 \mathrm{~mm})$ foi subdividida em cinco classes: muito grossa (2,0 a 1,0 mm), grossa ( 1,0 a $0,5 \mathrm{~mm})$, média $(0,5$ a $0,25 \mathrm{~mm})$, fina $(0,25$ a $0,105 \mathrm{~mm})$, e muito fina $(0,105$ a $0,053 \mathrm{~mm}$ ).

A densidade do solo (Ds) foi determinada em amostras com estrutura indeformada, segundo Blake \& Hartge (1986a), e a densidade de partículas (Dp) foi determinada pelo método do balão volumétrico, descrito por Blake \& Hartge (1986b). O volume total de poros (VTP) foi determinado segundo Danielson \& Sutherland (1986).

Os valores de argila total (AT) e argila dispersa em água (ADA), obtidos pela análise granulométrica, segundo metodologia preconizada pela Embrapa (1979), foram utilizados para determinação do índice de floculação (IF).

A estabilidade de agregados foi determinada por tamizamento em água, após pré-umedecimento lento, segundo Oliveira et al. (1983) e Kemper \& Rosenau (1986). A partir desta análise, foi determinada a proporção de agregados estáveis menores que $0,25 \mathrm{~mm}$, também chamada de índice de instabilidade, segundo El-Swaify \& Dangler (1977). O diâmetro médio geométrico (DMG) dos agregados foi calculado segundo Kemper \& Chepil (1965).

Na terra fina secada ao ar (TFSA) foram feitas análises de óxidos $\left(\mathrm{SiO}_{2}, \mathrm{Al}_{2} \mathrm{O}_{3}\right.$ e $\left.\mathrm{Fe}_{2} \mathrm{O}_{3}\right)$ extraídos pelo ataque sulfúrico, segundo Embrapa (1979). Na fração argila foram feitas determinações de $\mathrm{SiO}_{2}, \mathrm{Al}_{2} \mathrm{O}_{3}$ e $\mathrm{Fe}_{2} \mathrm{O}_{3}$ extraídos pelo ditionito-citrato-bicarbonato de sódio (DCB) (Mehra \& Jackson, 1960) e oxalato de amônio ácido (Schwertmann, 1964), objetivando-se obter os teores de óxidos livres totais e de óxidos menos cristalinos, respectivamente. Determinaram-se também $\mathrm{Al}_{2} \mathrm{O}_{3}$ e $\mathrm{Fe}_{2} \mathrm{O}_{3}$ extraídos pelo pirofosfato de sódio, objetivando-se obter os teores de óxidos complexados com a fração orgânica do solo (Sheldrick \& McKeague, 1975). A determinação do $\mathrm{Fe}_{2} \mathrm{O}_{3}$ foi feita por absorção atômica. $\mathrm{O} \mathrm{Al}_{2} \mathrm{O}_{3}$ foi determinado por colorimetria, pelo método do aluminon (Mckeague, 1978), sendo a eliminação do citrato obtida através da com- binação dos métodos de Mckeague (1978) e Camargo et al. (1986). A determinação do $\mathrm{SiO}_{2}$ foi feita por colorimetria, segundo Weaver et al. (1968). Todos os valores determinados na fração argila foram corrigidos para terra fina seca em estufa (TFSE) e todas as análises foram feitas com quatro repetições.

Os componentes do complexo sortivo, $\mathrm{pH}$ em água e em KCl $1 \mathrm{~N}$, e carbono orgânico foram determinados segundo Embrapa (1979).

$\mathrm{Na}$ fração areia, foi realizada a separação de minerais leves e pesados, por meio do tetrabromoetano, e determinada a relação entre esses minerais (Marshall \& Haseman, 1942).

Usou-se o programa estatístico SAS/STAT ${ }^{\mathrm{TM}}$ (SAS Institute, 1995), e os modelos foram ajustados por meio de análise de regressão linear múltipla, pelo processo "backward". O nível mínimo de significância pelo teste t para exclusão de uma variável no modelo foi de $\mathrm{P}>0,10$, o qual foi estipulado para preservação do nível global de significância do modelo (Draper \& Smith, 1981). Esse procedimento foi repetido até que não houvesse mais variáveis não-significativas.

\section{RESULTADOS E DISCUSSÃO}

Inicialmente, procurou-se correlacionar os valores de erodibilidade obtidos de maneira direta de todos os 19 solos envolvidos no estudo, com as variáveis morfológicas, físicas, químicas, mineralógicas e relações destas, que corresponderam a um total de 129 variáveis. Desse total, poucas foram as variáveis que apresentaram correlações lineares simples significativas com a erodibilidade. Tal resultado corrobora as observações de Wischmeier \& Mannering (1969), Obi et al. (1989) e Denardin (1990). Uma explicação para isso pode ser o fato de que a erodibilidade é um fator complexo que depende da interação de atributos múltiplos. Analisando as variáveis estudadas, fica difícil avaliar as relações de causa e efeito entre elas e os valores de erodibilidade, aspectos também salientados por Wischmeier \& Mannering (1969) e Denardin (1990), os quais ressaltam que a erodibilidade é uma variável que depende tanto da capacidade de infiltração da água no solo quanto de sua capacidade de resistir à desagregação e ao transporte provocados pela chuva e pelo escorrimento superficial.

Pesq. agropec. bras., Brasília, v.34, n.12, p.2287-2298, dez. 1999 
Assim, procurou-se relacionar a erodibilidade (Y) com as variáveis determinadas pela análise de regressão linear múltipla, pelo processo "backward"; das 129 variáveis testadas, 37 (Tabela 1) tomaram parte em pelo menos um dos seguintes modelos:

Modelo 1: $\mathrm{Y}=4,77 \times 10^{-2}-9,66 \times 10^{-3} \mathrm{X}_{14}+1,63 \times 10^{-2} \mathrm{X}_{16}$ -
$1,12 \times 10^{-2} \mathrm{X}_{17}+1,85 \times 10^{-2} \mathrm{X}_{18}-1,51 \times 10^{-2} \mathrm{X}_{19}-2,46 \times 10^{-4} \mathrm{X}_{22}$ $-3,58 \times 10^{-4} X_{23}+1,47 \times 10^{-4} X_{24}-1,43 \times 10^{-4} X_{25}+3,26 \times 10^{-3} X_{26}$ $-1,26 \times 10^{-3} \mathrm{X}_{27}-2,29 \times 10^{-4} \mathrm{X}_{31}+1,07 \times 10^{-4} \mathrm{X}_{32}+2,69 \times 10^{-4} \mathrm{X}_{34}$, $\mathrm{r}^{2}=0,98 * *$

Modelo 2: $\mathrm{Y}=0,112+2,50 \times 10^{-3} \mathrm{X}_{20}-7,07 \times 10^{-4} \mathrm{X}_{21}$ $+7,96 \times 10^{-5} X_{22}-1,01 \times 10^{-4} X_{25}+1,56 \times 10^{-3} X_{26}$ - $3,34 \times 10^{-4} X_{27}-1,48 \times 10^{-4} X_{28}-1,55 \times 10^{-4} X_{29}-1,67 \times 10^{-4} X_{30}$ $+5,30 \times 10^{-5} \mathrm{X}_{34}-1,24 \times 10^{-3} \mathrm{X}_{35}, \mathrm{r}^{2}=0,92^{* *}$

TABELA 1. Descrição, códigos, valores mínimos, médios e máximos das variáveis dos solos estudados incluídas em modelos para estimativa da erodibilidade em latossolos brasileiros ${ }^{1}$.

\begin{tabular}{|c|c|c|c|c|}
\hline \multirow[t]{2}{*}{ Variável } & \multirow[t]{2}{*}{ Descrição } & \multicolumn{3}{|c|}{ Valor } \\
\hline & & Mínimo & Médio & Máximo \\
\hline K obs. & Erodibilidade $\left(\mathrm{t} \mathrm{h} \mathrm{MJ}^{-1} \mathrm{~mm}^{-1}\right)$ & 0,002 & 0,011 & 0,034 \\
\hline $\mathrm{X}_{1}$ & Relação entre minerais leves e pesados na fração areia (adimensional) & 0,13 & 0,97 & 1,00 \\
\hline $\mathrm{X}_{2}$ & Capacidade de troca de cátions do solo a pH 7,0 $\left(\mathrm{cmol}_{\mathrm{c}} \mathrm{kg}^{-1}\right)$ & 4,1 & 8,6 & 18,1 \\
\hline $\mathrm{X}_{3}$ & Teor de matéria orgânica $\left(\mathrm{g} \mathrm{kg}^{-1}\right)$ & 9,0 & 26,0 & 47,0 \\
\hline $\mathrm{X}_{4}$ & $\Delta \mathrm{pH}=\mathrm{pH} \mathrm{KCl}-\mathrm{pH} \mathrm{H} \mathrm{H}_{2} \mathrm{O}$ (adimensional) & $-0,4$ & $-0,7$ & $-1,3$ \\
\hline $\mathrm{X}_{5}$ & Teor de matéria orgânica multiplicado pelo $\Delta \mathrm{pH}\left(\mathrm{g} \mathrm{kg}^{-1}\right)$ & $-4,5$ & $-15,2$ & $-42,0$ \\
\hline $\mathrm{X}_{6}$ & $\mathrm{Al}_{2} \mathrm{O}_{3 \text { (d) }}$ extraído pelo DCB $\left(\mathrm{g} \mathrm{kg}^{-1}\right)$ & 3,0 & 18,0 & 35,0 \\
\hline $\mathrm{X}_{7}$ & $\mathrm{Al}_{2} \mathrm{O}_{3}$ (o) extraído pelo oxalato de amônio $\left(\mathrm{g} \mathrm{kg}^{-1}\right)$ & 2,0 & 9,0 & 94,0 \\
\hline $\mathrm{X}_{8}$ & $\mathrm{Al}_{2} \mathrm{O}_{3}$ (p) extraído pelo pirofosfato de sódio $\left(\mathrm{g} \mathrm{kg}^{-1}\right)$ & 0,10 & 0,50 & 1,20 \\
\hline $\mathrm{X}_{9}$ & $\mathrm{SiO}_{2 \text { (d) }}$ extraído pelo DCB $\left(\mathrm{g} \mathrm{kg}^{-1}\right)$ & 2,0 & 7,0 & 12,0 \\
\hline $\mathrm{X}_{10}$ & Relação $\left(\mathrm{Fe}_{2} \mathrm{O}_{3}\right.$ (o) $\left.+\mathrm{Al}_{2} \mathrm{O}_{3 \text { (o) }}\right) / \mathrm{SiO}_{2 \text { (o) }}$ (adimensional) & 1,5 & 7,5 & 34,7 \\
\hline $\mathrm{X}_{11}$ & Relação $\mathrm{Fe}_{2} \mathrm{O}_{3(\mathrm{p} /} / \mathrm{Al}_{2} \mathrm{O}_{3}$ (p) (adimensional) & 0,50 & 4,3 & 14,0 \\
\hline $\mathrm{X}_{12}$ & Relação $\mathrm{Fe}_{2} \mathrm{O}_{3}$ (o) $/ \mathrm{SiO}_{2}$ (o) (adimensional) & 0,5 & 2,0 & 5,5 \\
\hline $\mathrm{X}_{13}$ & Relação Ki (adimensional) & 0,67 & 1,33 & 2,03 \\
\hline $\mathrm{X}_{14}$ & Código do matiz do solo úmido segundo Munsell (adimensional) & 1 & 3 & 4 \\
\hline$X_{15}$ & Código da drenagem do perfil do solo (adimensional) & 2 & 3 & 5 \\
\hline $\mathrm{X}_{16}$ & Código do grau da estrutura (adimensional) & 2 & 3 & 4 \\
\hline $\mathrm{X}_{17}$ & Código do tamanho da estrutura (adimensional) & 1,5 & 2,5 & 4 \\
\hline $\mathrm{X}_{18}$ & Código da forma da estrutura (adimensional) & 2 & 2,5 & 3 \\
\hline $\mathrm{X}_{19}$ & Código da plasticidade do solo (adimensional) & 2 & 3 & 4 \\
\hline $\mathrm{X}_{20}$ & Teor de AMG dispersa com NaOH 0,1 mol L-1 $\left(\mathrm{g} \mathrm{kg}^{-1}\right)$ & 0 & 2 & 30 \\
\hline $\mathrm{X}_{21}$ & Teor de AG dispersa com NaOH $0,1 \mathrm{~mol} \mathrm{~L}^{-1}\left(\mathrm{~g} \mathrm{~kg}^{-1}\right)$ & 0 & 20 & 150 \\
\hline $\mathrm{X}_{22}$ & Teor de AF dispersa com NaOH $0,1 \mathrm{~mol} \mathrm{~L}^{-1}\left(\mathrm{~g} \mathrm{~kg}^{-1}\right)$ & 0 & 120 & 397 \\
\hline $\mathrm{X}_{23}$ & Teor de AMF dispersa com NaOH $0,1 \mathrm{~mol} \mathrm{~L}^{-1}\left(\mathrm{~g} \mathrm{~kg}^{-1}\right)$ & 7 & 50 & 490 \\
\hline$X_{24}$ & Teor de silte disperso com $\mathrm{NaOH} 0,1 \mathrm{~mol} \mathrm{~L}^{-1}\left(\mathrm{~g} \mathrm{~kg}^{-1}\right)$ & 1 & 80 & 160 \\
\hline $\mathrm{X}_{25}$ & Teor de argila dispersa com $\mathrm{NaOH} 0,1 \mathrm{~mol} \mathrm{~L}^{-1}\left(\mathrm{~g} \mathrm{~kg}^{-1}\right)$ & 139 & 600 & 860 \\
\hline $\mathrm{X}_{26}$ & Teor de AMG dispersa em água $\left(\mathrm{g} \mathrm{kg}^{-1}\right)$ & 0 & 10 & 35 \\
\hline $\mathrm{X}_{27}$ & Teor de AG dispersa em água $\left(\mathrm{g} \mathrm{kg}^{-1}\right)$ & 0 & 60 & 160 \\
\hline $\mathrm{X}_{28}$ & Teor de AM dispersa em água $\left(\mathrm{g} \mathrm{kg}^{-1}\right)$ & 50 & 130 & 230 \\
\hline $\mathrm{X}_{29}$ & Teor de AF dispersa em água $\left(\mathrm{g} \mathrm{kg}^{-1}\right)$ & 10 & 192 & 670 \\
\hline $\mathrm{X}_{30}$ & Teor de AMF dispersa em água $\left(\mathrm{g} \mathrm{kg}^{-1}\right)$ & 60 & 90 & 480 \\
\hline$X_{31}$ & Teor de silte disperso em água $\left(\mathrm{g} \mathrm{kg}^{-1}\right)$ & 60 & 240 & 629 \\
\hline $\mathrm{X}_{32}$ & Teor de argila dispersa em água $\left(\mathrm{g} \mathrm{kg}^{-1}\right)$ & 1 & 170 & 330 \\
\hline $\mathrm{X}_{33}$ & Volume total de poros $\left(\mathrm{dm}^{3} \mathrm{dm}^{-3}\right)$ & 0,40 & 0,53 & 0,65 \\
\hline $\mathrm{X}_{34}$ & Índice de floculação (adimensional) & 0,555 & 0,685 & 0,999 \\
\hline $\mathrm{X}_{35}$ & Diâmetro médio geométrico de agregados (mm) & 1,46 & 3,11 & 4,35 \\
\hline $\mathrm{X}_{36}$ & Índice de instabilidade de agregados $\left(\mathrm{g} \mathrm{kg}^{-1}\right)$ & 21,5 & 72,4 & 313,9 \\
\hline \multirow[t]{2}{*}{$\mathrm{X}_{37}$} & Relação entre $(\mathrm{AMF}+$ Silte $) /(\mathrm{AMG}+\mathrm{AG}+\mathrm{AM}+\mathrm{AF})$ & & & \\
\hline & dispersos em água (adimensional) & 0,20 & 0,80 & 3,00 \\
\hline
\end{tabular}

$1 \Delta \mathrm{pH}=$ diferença entre $\mathrm{pH} \mathrm{KCI}$ e $\mathrm{pH} \mathrm{H} \mathrm{H}_{2} \mathrm{O} ; \mathrm{pH} \mathrm{KCI}=\mathrm{pH}$ determinado em solução de cloreto de potássio; $\mathrm{pH} \mathrm{H}_{2} \mathrm{O}=$ pH determinado em solução de água; $\mathrm{DCB}=$ ditionito citrato bicarbonato; $\mathrm{Ki}=$ relação molecular entre $\mathrm{SiO}_{2}$ e $\mathrm{Al}_{2} \mathrm{O}_{3} ; \mathrm{AMG}=$ areia muito grossa; $\mathrm{AG}=$ areia grossa; $\mathrm{AF}=$ areia fina; $\mathrm{AMF}=$ areia muito fina; $\mathrm{AM}=$ areia média. 
Modelo 3: $\mathrm{Y}=-3,89 \times 10^{-2}+5,11 \times 10^{-3} \mathrm{X}_{14}-1,25 \times 10^{-2} \mathrm{X}_{15}$ $+5,41 \times 10^{-3} \mathrm{X}_{16}-7,27 \times 10^{-3} \mathrm{X}_{18}+5,33 \times 10^{-2} \mathrm{X}_{33}+3,21 \times 10^{-5} \mathrm{X}_{34}$ - $5,66 \times 10^{-5} X_{36}+8,33 \times 10^{-4} X_{2}-1,17 \times 10^{-2} X_{4}+$ $1,53 \times 10^{-2} \mathrm{X}_{13}, \mathrm{r}^{2}=0,91 * *$

Modelo 4: $\mathrm{Y}=7,87 \times 10^{-2}-4,99 \times 10^{-4} \mathrm{X}_{3}+5,41 \times 10^{-2} \mathrm{X}_{8}$ - $3,21 \times 10^{-3} X_{9}-6,29 \times 10^{-3} X_{15}+2,53 \times 10^{5} X_{34}-9,56 \times 10^{-3} X_{35}$ $-1,41 \times 10^{-4} X_{36}-9,29 \times 10^{-3} X_{37}, r^{2}=0,83^{* *}$

Modelo 5: $\mathrm{Y}=-3,21 \times 10^{-2}-4,26 \times 10^{-2} \mathrm{X}_{4}-8,65 \times 10^{-4} \mathrm{X}_{6}$ $+9,51 \times 10^{-4} X_{7}+3,13 \times 10^{-2} X_{8}+2,65 \times 10^{-2} X_{1}+$ $8,83 \times 10^{-4} \mathrm{X}_{5}-2,32 \times 10^{-3} \mathrm{X}_{10}+3,39 \times 10^{-3} \mathrm{X}_{11}+$ $4,15 \times 10^{-3} \mathrm{X}_{12}, \mathrm{r}^{2}=0,72^{*}$

Embora não exista relação de causa e efeito entre as variáveis selecionadas e a erodibilidade, fica claro que a maioria delas tem participação nos processos que governam a erosão, e não são, no seu conjunto, muito diferentes das selecionadas em outros estudos (Wischmeier \& Mannering, 1969; Wischmeier et al., 1971; El-Swaify \& Dangler, 1977; Römkens et al., 1977; Denardin, 1990).

Os modelos apresentaram elevados coeficientes de determinação e apresentaram a mesma magnitude que os encontrados em outros trabalhos (Wischmeier et al., 1971; El-Swaify \& Dangler, 1977; Römkens et al., 1977; Denardin, 1990). A complexidade das equações é apenas aparente, pois sua linearidade facilita muito os cálculos. Além disso, nos modelos de previsão de perdas de solo são utilizados, atualmente, recursos computacionais, o que reduz o trabalho de cálculo.

Embora as equações geradas necessitem de um número considerável de variáveis, estas são, sem exceção, de fácil determinação ou prontamente disponíveis nos boletins de levantamentos de solos. É possível que apenas os óxidos extraídos por ditionito-citrato-bicarbonato de sódio, oxalato de amônio ácido e pirofosfato de sódio e relação de minerais leves e pesados presentes na fração areia não sejam rotineiramente determinados na maioria dos laboratórios de análise de solo nacionais. A escolha do modelo a ser empregado pode ser feita com base no coeficiente de determinação, na disponibilidade de variáveis, e em função da precisão desejada para a determinação da erodibilidade. $\mathrm{O}$ uso dessas equações restringe-se a latossolos cujos atributos não possuam valores fora das amplitudes apresentadas na Tabela 1 .

Com relação aos atributos de campo, a cor do solo expressa pelo matiz foi considerada nos modelos 1 e 3 . Houve uma tendência de os solos com matiz 10R e 2,5YR (mais avermelhados) apresentarem maiores valores de erodibilidade que os com matiz 10YR (mais amarelados), com exceção do LV (Latossolo Vermelho-Amarelo) de Ubajára, CE. Estes valores mais elevados de erodibilidade apresentados pelos solos mais avermelhados, talvez estejam associados à mais baixa coerência (coesão) entre agregados nestes solos, e favoreçam o arraste deles (Resende, 1985). Por outro lado, Lima et al. (1990) estimaram a erodibilidade por métodos indiretos e encontraram resultados inversos em latossolos pertencentes a uma mesma região, ou seja, os solos mais avermelhados apresentaram maior resistência à erosão, devido, segundo os autores, ao maior arredondamento e menor tamanho da estrutura granular, favorecendo a permeabilidade. Novamente o balanço entre estes processos é fundamental no entendimento do comportamento destes solos em face do processo erosivo.

Dos componentes caracterizadores da estrutura do solo (tamanho, grau e tipo), todos foram incluídos nos modelos 1 e 3, com exceção do tamanho. Nos trabalhos de Silva et al. (1994, 1995), verificouse que solos com unidades estruturais menores, embora apresentassem maior quantidade de solo salpicada, possuíam menor erodibilidade. É possível que, as partículas salpicadas desses solos fossem constituídas das próprias unidades estruturais que, com o salpico, apenas mudavam de posição, sem alterar a estrutura da camada superficial, sem redução da infiltração. O tamanho das unidades estruturais do horizonte A pode ser facilmente determinado no campo (Lemos \& Santos, 1996) ou obtido por meio das informações constantes na descrição morfológica dos perfis dos solos.

A combinação de atributos morfoestruturais é importante para o estudo da erosão hídrica. No presente estudo, de modo geral, houve uma tendência de os solos com estrutura forte a moderada muito 
pequena a pequena granular estarem relacionados com os maiores valores de erodibilidade. Estes resultados estão de acordo com as considerações de Resende et al. (1997), os quais comentam que latossolos com estes atributos estruturais são facilmente arrastados pela enxurrada proveniente das chuvas, fazendo com que a erodibilidade atinja valores mais elevados. Entretanto, estas interpretações devem ser feitas com cautela, pois solos que apresentam atributos estruturais favoráveis, ou seja, tamanho pequeno e baixa coesão entre partículas ou agregados, em alguns casos podem apresentar baixa erodibilidade, caso a permeabilidade seja elevada. Estas observações corroboram estudos de Lima et al. (1990) e Silva et al. (1994, 1995).

Quanto à consistência do solo avaliada em campo, em diversos graus de umidade, a plasticidade foi a única variável incluída no modelo 1. De modo geral, os resultados encontrados para a consistência estão condizentes com as faixas de erodibilidade apresentadas pelos solos. Dentre as oito classes de drenagem inferidas a campo (Lemos \& Santos, 1996), no presente estudo foram consideradas apenas seis, que foram incluídas nos modelos 3 e 4 .

A distribuição do tamanho de partículas e agregados obtida com dispersão total ( $\left.\mathrm{NaOH} 0,1 \mathrm{~mol} \mathrm{~L}^{-1}\right)$ e em água, bem como relações destas, foram incluídas nos modelos 1, 2, 3 e 4 . O índice de floculação (Tabela 1) foi incluído em todos os modelos citados. No modelo 1 foram incluídas a argila e silte com dispersão total e em água; a areia fina (AF) e areia muito fina (AMF) com dispersão total, e a areia muito grossa (AMG) e areia grossa (AG) com dispersão em água (Tabela 1). No modelo 2 foram incluídas a AMG, AG e AF com dispersão total e em água; a argila com dispersão total e a areia média (AM) e AMF com dispersão em água (Tabela 1). No modelo 4 foi incluída a relação entre a porcentagem de AMF somada ao silte e a soma de AMG, AG, AM e AF (Tabela 1). A distribuição do tamanho de partículas e agregados obtida com dispersão total $(\mathrm{NaOH}$ $0,1 \mathrm{~mol} \mathrm{~L}^{-1}$ ) e em água é utilizada em uma série de modelos estimativos da erodibilidade; entre esses, pode-se citar os trabalhos de Dumas (1965), Wischmeier \& Mannering (1969), Wischmeier et al. (1971), Roth et al. (1974), Lombardi Neto \& Bertoni (1975), El-Swaify \& Dangler (1977), Römkens et al. (1977), Denardin (1990), Lima et al. (1990), Paez \& Ildefonso (1992), Chaves (1994) e Marques el al. (1997b). A porosidade total do solo foi incluída no modelo 3.

Dos índices que expressam a estabilidade de agregados, o diâmetro médio geométrico (DMG) foi incluído nos modelos 2 e 4, e os agregados menores que $0,25 \mathrm{~mm}$, chamado de índice de instabilidade por El-Swaify \& Dangler (1977), foram incluídos nos modelos 3 e 4 . Outros autores também consideraram esses índices em estudos semelhantes, entre os quais pode-se destacar Wischmeier \& Mannering (1969), El-Swaify \& Dangler (1977) e Angulo (1983). Segundo Le Bissonnais (1996), os solos podem ser separados de acordo com o DMG, e os solos com DMG maior que $2 \mathrm{~mm}$ foram classificados como muito estáveis e resistentes à erosão. Tais observações estão de acordo com os resultados encontrados no presente estudo, onde apenas dois solos (Latossolos Roxo de Londrina e de Dourados) apresentaram valores de DMG menores que $2 \mathrm{~mm}$, e a erodibilidade desses solos foi das mais elevadas para o conjunto dos latossolos estudados. Do ponto de vista de estabilidade de agregados, os resultados sugerem que, quanto maiores os agregados, menor é a erodibilidade do solo. A inclusão desses índices nos modelos é importante, do ponto de vista de manejo, uma vez que eles podem facilmente ser alterados, dependendo do manejo a que o solo está sendo submetido.

Em relação às variáveis químicas, os óxidos $\left(\mathrm{Fe}_{2} \mathrm{O}_{3}, \mathrm{Al}_{2} \mathrm{O}_{3}\right.$ e $\left.\mathrm{SiO}_{2}\right)$ extraídos pelo ataque sulfúrico, DCB, oxalato de amônio ácido e pirofosfato de sódio e relações destes também fazem parte dos modelos 3, 4 e 5. No modelo 3, foi incluída a relação $\mathrm{Ki}\left(\mathrm{Al}_{2} \mathrm{O}_{3} / \mathrm{SiO}_{2}\right)$; segundo Marques et al. (1997a), os solos com valores mais baixos de $\mathrm{Ki}$ foram os que apresentaram maior correlação com o modelo para estimativa da erodibilidade adaptado por Lima et al. 
(1990). No modelo 4, foram incluídos o $\mathrm{Al}_{2} \mathrm{O}_{3}$ extraído pelo pirofosfato de sódio e $\mathrm{SiO}_{2}$ extraído pelo DCB. No modelo 5, foram incluídos o $\mathrm{Al}_{2} \mathrm{O}_{3}$ extraído pelo DCB, o oxalato de amônio ácido e o pirofosfato de sódio, e as relações $\left(\mathrm{Fe}_{2} \mathrm{O}_{3}+\mathrm{Al}_{2} \mathrm{O}_{3}\right) /$ $\mathrm{SiO}_{2}$ e $\mathrm{Fe}_{2} \mathrm{O}_{3} / \mathrm{SiO}_{2}$, extraídas pelo oxalato de amônio ácido, e $\mathrm{Fe}_{2} \mathrm{O}_{3} / \mathrm{Al}_{2} \mathrm{O}_{3}$ extraídos pelo pirofosfato de sódio (Tabela 1). É possível que essas formas atuem na microagregação de solos mais intemperizados (Uehara \& Gillman, 1981), podendo estar contribuindo para a elevação ou redução da erodibilidade. Elas estão associadas à agregação do solo por meio da interação com óxidos de ferro e de alumínio, e contribuem para redução da coesão entre agregados e aumento da erodibilidade, por este aspecto, embora tenda a ocorrer um aumento da permeabilidade nesse mesmo sentido (Resende, 1985). Por outro lado, a interação entre os óxidos de silício, ferro e alumínio com maior reatividade, talvez esteja aumentando a coesão e contribuindo para a redução da erodibilidade. $\mathrm{O}$ balanço entre estes processos passa a ser decisivo.

O valor de $\Delta \mathrm{pH}$ está presente nos modelos 3 e 5 . Com a elevação do $\mathrm{pH}$, há uma predisposição para a elevação dos valores de argila dispersa em água, afetando significativamente os atributos importantes do solo relacionados ao processo erosivo, como: encrostamento, agregação, porosidade, infiltração de água e arraste de partículas e agregados pela enxurrada, podendo estar, desta forma, contribuindo para um aumento ou redução da erodibilidade (Uehara \& Gillman, 1981; Angulo, 1983). A matéria orgânica, presente no modelo 4, certamente contribui para que os valores de erodibilidade permaneçam na faixa de valores classificados em muito baixos e baixos (Freire \& Pessotti, 1974). Segundo Wischmeier \& Mannering (1969), a significância da correlação entre erodibilidade e matéria orgânica decresce substancialmente em solos argilosos e muito argilosos.

Entre as variáveis mineralógicas estudadas, a caracterização quantitativa da fração areia, realizada em função da relação entre minerais leves e pesados, está presente no modelo 5. Esses resultados pressupõem que a presença de minerais pesados $\left(\mathrm{d} \geq 2,97 \mathrm{~g} \mathrm{~cm}^{-3}\right)$, na fração areia do solo, possibilita um menor arraste destes pela água da chuva, e propicia menores valores de erodibilidade. Resultados semelhantes foram observados em estudos desenvolvidos por Silva et al. (1994), em Latossolo Vermelho-Escuro e Latossolo Vermelho-Amarelo sob cerrado.

Nas Figs. 1 e 2 estão reunidos, respectivamente, os gráficos dos resíduos padronizados e a dispersão dos pontos da erodibilidade observada e estimada pelos modelos. Observa-se que a distribuição dos resíduos padronizados está dentro da faixa 2 e -2, o que indica que esses resíduos são aceitáveis (Draper \& Smith, 1981). Como os erros estão uniformemente distribuídos ao longo dos valores da erodibilidade estimada, pode-se afirmar que não existe tendência a super ou subestimação destes valores.

Todos os modelos propostos para estimativa da erodibilidade podem ser utilizados, mas o modelo 1 foi escolhido por ser, entre os de mais alto $r^{2}$, o que possui variáveis de mais fácil determinação no campo (matiz, grau, tamanho e tipo da estrutura, e plasticidade) e variáveis determinadas rotineiramente nos laboratórios, como análise granulométrica do solo (areia fina, areia muito fina, silte e argila), fracionamento da terra fina em água (frações correspondentes à areia muito grossa, areia grossa, silte e argila) e índice de floculação. Os modelos 3 e 4 reúnem variáveis que podem ser alteradas com o manejo do solo, como: capacidade de troca de cátions, matéria orgânica, $\Delta \mathrm{pH}$, drenagem, estrutura, plasticidade, porosidade, índice de floculação, diâmetro médio geométrico dos agregados e índice de estabilidade de agregados (Tabela 1). Portanto, os modelos 3 e 4 podem ser utilizados para monitoramento da suscetibilidade do solo à erosão quando sob manejo, tornando-se um indicativo da qualidade do solo em relação ao processo erosivo (Karlen \& Stott, 1994), contribuindo para a sustentabilidade ambiental. 

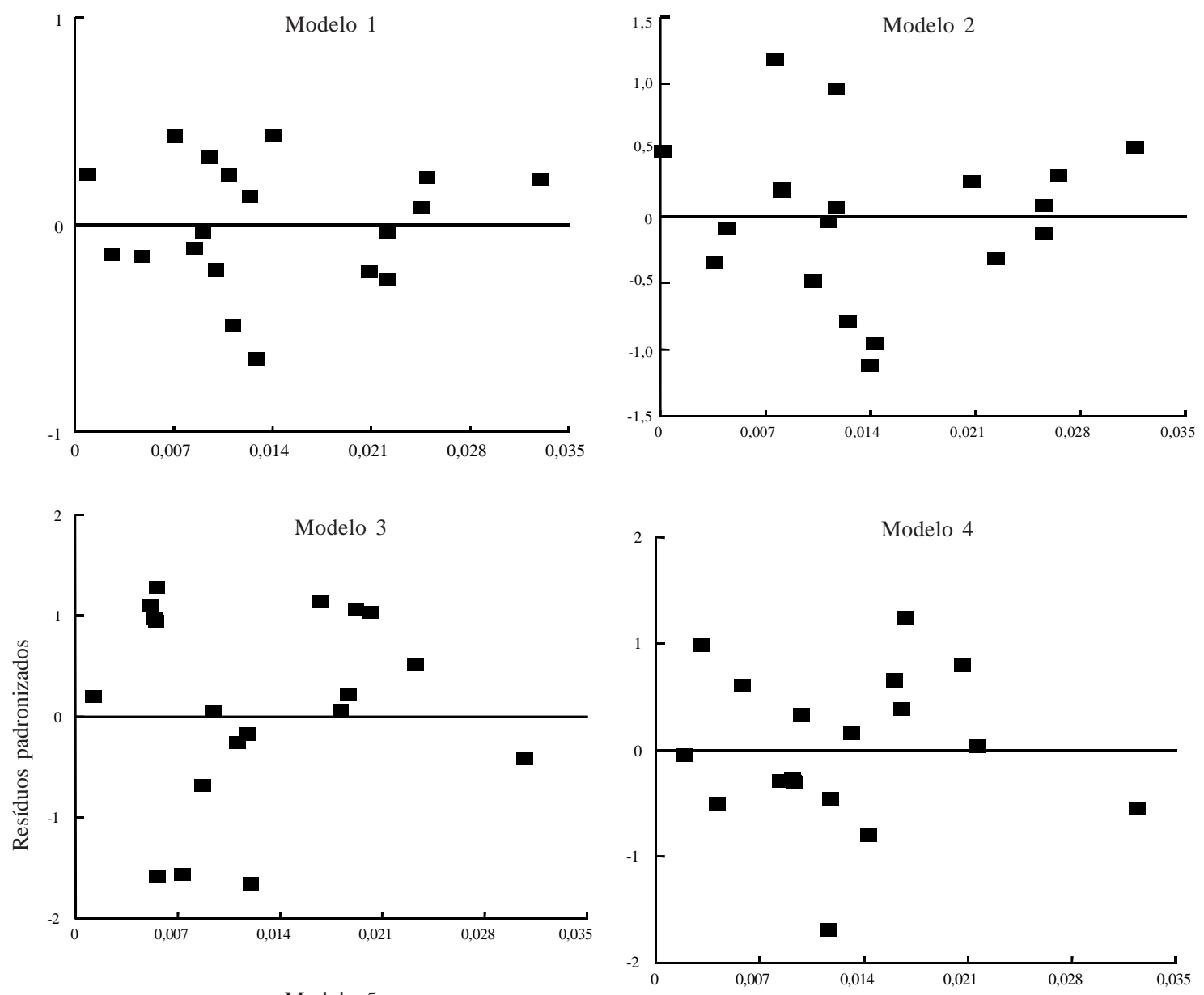

Modelo 5

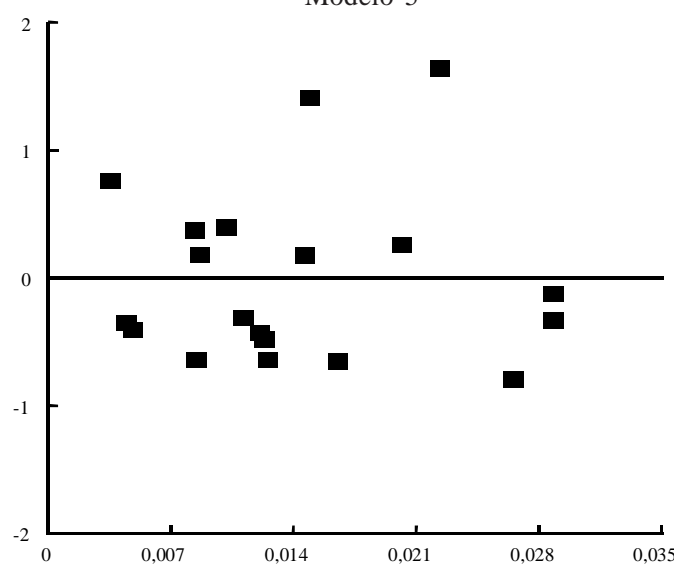

Erodibilidade estimada $\left(\mathrm{t} \mathrm{h}(\mathrm{MJ} \mathrm{mm})^{-1}\right)$

- Dispersão da Relação

Erodibilidade estimada $\left(\mathrm{t} \mathrm{h}(\mathrm{MJ} \mathrm{mm})^{-1}\right)$

FIG. 1. Distribuição dos resíduos padronizados da erodibilidade estimada pelos modelos propostos. 

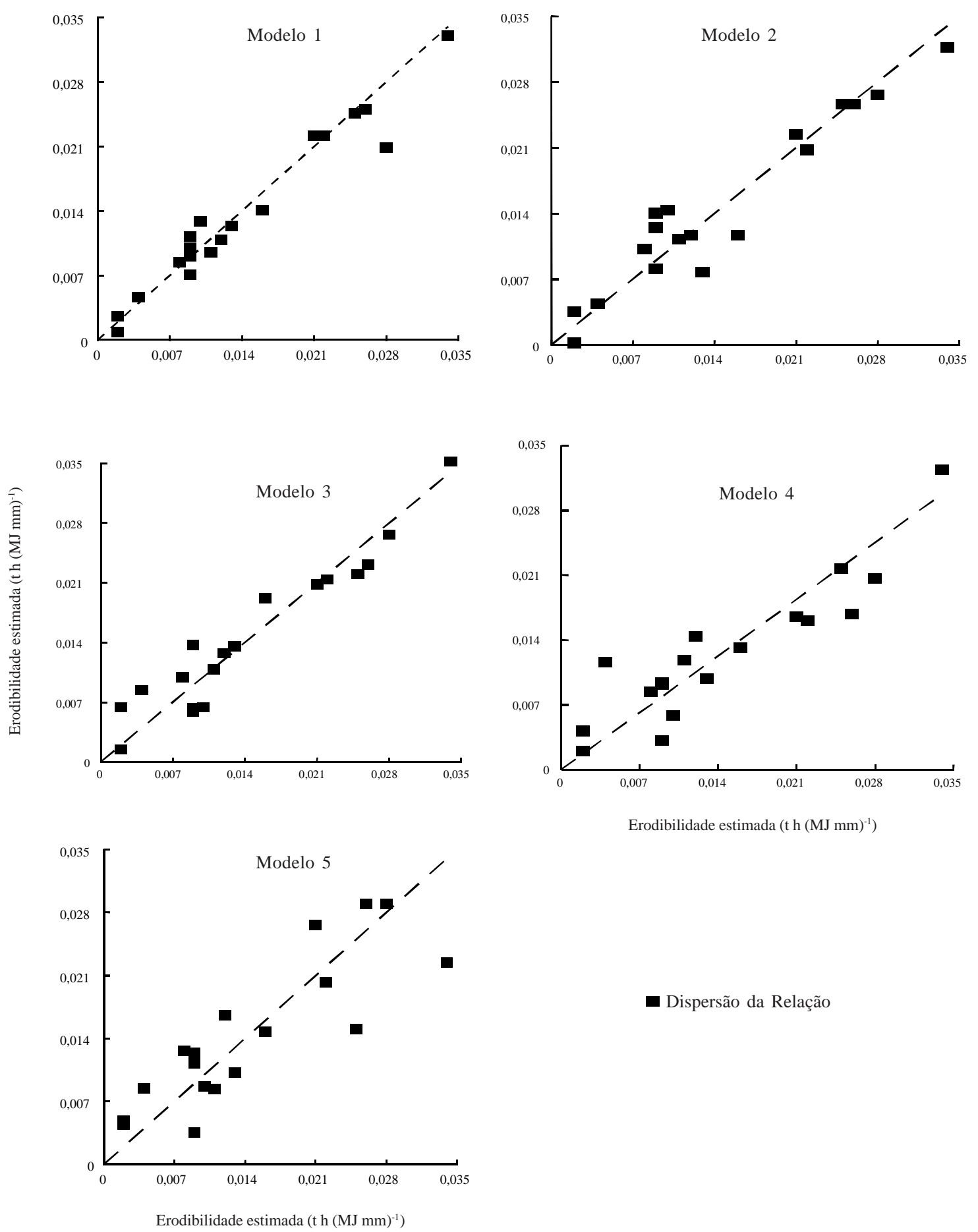

- Dispersão da Relação

FIG. 2. Relação entre os valores observados e estimados da erodibilidade pelos modelos propostos. 


\section{CONCLUSÃO}

Os cinco modelos propostos apresentam bom desempenho para a estimativa da erodibililidade dos latossolos brasileiros, notadamente o modelo 1, por ser, entre os de mais alto $\mathrm{r}^{2}$, o que possui variáveis de mais fácil determinação no campo e variáveis determinadas rotineiramente nos laboratórios.

\section{REFERÊNCIAS}

ANGULO, J.R. Relações entre a erodibilidade e algumas propriedades de solos brasileiros. Curitiba: UFPR, 1983. 154p. Dissertação de Mestrado.

BLAKE, G.R.; HARTGE, K.H. Bulk density. In: KLUTE, A. (Ed.). Methods of soil analysis. 2.ed. Madison: American Society of Agronomy, 1986a. v.1, p.363375.

BLAKE, G.R.; HARTGE, K.H. Partycle density. In: KLUTE, A. (Ed.). Methods of soil analysis. 2.ed. Madison: American Society of Agronomy, 1986b. v.1, p.377-382.

CAMARGO, O.A. de; MONIZ, A.C.; JORGE, J.A.; VALADARES, J.M.A.S. Métodos de análise química, mineralógica e física de solos do Instituto Agronômico de Campinas. Campinas: IAC, 1986. 94p. (Boletim técnico, 106).

CHAVES, H.M.L. Estimativa da erosão atual e potencial no Vale do Rio S. Francisco. Brasília: FAO, 1994. 34p. Relatório 2257.

DANIELSON, R.E.; SUTHERLAND, P.L. Porosity. In: KLUTE, A. (Ed.). Methods of soil analysis. 2.ed. Madison: American Society of Agronomy, 1986. v.1, p.443-461.

DAY, P.R. Particle fractionation and particle-size analysis. In: BLACK, C.A. (Ed.). Methods of soil analysis. Madison: American Society of Agronomy, 1965. v.1, p.545-566. (Agronomy, 9).

DENARDIN, J.E. Erodibilidade de solo estimada por meio de parâmetros físicos e químicos. Piracicaba: ESALQ, 1990. 81p. Tese de Doutorado.

DRAPER, N.R.; SMITH, H. Applied regression analysis. 2.ed. New York: J. Wiley, 1981. 704p.

DUMAS, J. Relation entre l'érodibilité des sols et leurs caractéristiques analytiques. Cahiers Orstom: Serie Pédologie, Paris, v.3, n.4, p.307-333, 1965.

Pesq. agropec. bras., Brasília, v.34, n.12, p.2287-2298, dez. 1999
EL-SWAIFY, S.A.; DANGLER, E.W. Erodibilities of selected tropical soils in relation to structural and hydrologic parameters. In: NATIONAL CONFERENCE ON SOIL EROSION, 1976, West Lafayette. Proceedings. Ankeny: Soil Conservation Society of America, 1977. p.105-114.

EMBRAPA. Serviço Nacional de Levantamento e conservação de Solos (Rio de Janeiro, RJ). Manual de métodos de análise de solos. Rio de Janeiro, 1979. não paginado.

FREIRE, O.; PESSOTTI, J.E.S. Erodibilidade dos solos do Estado de São Paulo. Anais da Escola Superior de Agricultura "Luiz de Queiroz", Piracicaba, v.33, p.333-350, 1974.

HENKLAIN, J.C.; FREIRE, O. Avaliação do método nomográfico para determinação da erodibilidade de latossolos do Estado do Paraná. Revista Brasileira de Ciência do Solo, Campinas, v.7, n.2, p.191-195, maio/ago. 1983.

KARLEN, D.L.; STOTT, D.E. A framework for evaluating physical and chemical indicators of soil quality. In: DORAN, J.W.; COLEMAN, D.C.; BEZDICEK, D.F.; STEWART, B.A. (Eds.). Defining soil quality for a sustainable environment. Madison: Soil Science Society of America/American Society of Agronomy, 1994. p.53-72. (Special publication, 35).

KEMPER, W.D.; CHEPIL, W.S. Size distribution of aggregates. In: BLACK, C.A. (Ed.). Methods of soil analysis. Madison: American Society of Agronomy, 1965. Part. 1, p.499-509. (Agronomy, 9).

KEMPER, W.D.; ROSENAU, R.C. Aggregate stability and size distribution. In: KLUTE, A. (Ed.). Methods of soil analysis. Madison: American Society of Agronomy, 1986. v.1, p.425-442.

LE BISSONNAIS, Y. Aggregate stability and assessment of soil crustability and erodibility: I. theory and methodology. European Journal of Soil Science, Oxford, v.47, n.4, p.425-437, Dec. 1996.

LEMOS, R.C.; SANTOS, R.D. Manual de descrição e coleta de solo no campo. 3.ed. Campinas: Sociedade Brasileira de Ciência do Solo, 1996. 83p.

LIMA, J.M.; CURI, N.; RESENDE, M.; SANTANA, D.P. Dispersão do material de solo em água para avaliação indireta da erodibilidade em latossolos. Revista Brasileira de Ciência do Solo, Campinas, v.14, n.1, p.85-90, jan./abr. 1990. 
LOMBARDI NETO, F.; BERTONI, J. Erodibilidade de solos paulistas. Campinas: Instituto Agronômico, 1975. 12p. (Boletim técnico, 27).

MARQUES, J.J.G.S.M.; CURI, N.; FERREIRA, M.M.; LIMA, J.M.; SILVA, M.L.N.; SÁ, M.A.C. de. Adequação de métodos indiretos para estimativa da erodibilidade de solos com horizonte B textural no Brasil. Revista Brasileira de Ciência do Solo, Viçosa, v.21, n.3, p.447-456, 1997a.

MARQUES, J.J.G.S.M.; CURI, N.; LIMA, J.M.; FERREIRA, M.M.; SILVA, M.L.N.; FERREIRA, D.F. Estimativa da erodibilidade a partir de atributos de solos com horizonte B textural no Brasil. Revista Brasileira de Ciência do Solo, Viçosa, v.21, n.3, p.457-465, 1997b.

MARSHALL, C.E.; HASEMAN, J.F. The quantitative evaluation of soil formation and development by heavy mineral studies. Soil Science Society of America. Proceedings, Madison, v.7, p.448-453, 1942.

MARTINS FILHO, E.C.; SILVA, J.R.C. Comparação de métodos de avaliação da erodibilidade em Latossolo Vermelho-Amarelo distrófico. Revista Brasileira de Ciência do Solo, Campinas, v.9, n.2, p.175-177, maio/ago. 1985.

McKEAGUE, J.A. Manual on soil sampling and methods of analysis. 2.ed. Ottawa: Canadian Society of Soil Science, 1978. 212p.

MEHRA, O.P.; JACKSON, M.L. Iron oxide removal from soils and clays by a dithionite-citrate system buffered with sodium bicarbonate. Clays and Clay Minerals, Clarkson, v.3, p.317-327, 1960.

MUNSELL COLOR COMPANY. Munsell soil color charts. Baltimore, MD, 1975. n.p. Catálogo.

OBI, M.E.; SALAKO, F.K.; LAL, R. Relative susceptibility of some southeastern Nigeria soils to erosion. Catena, Cremlingen, v.16, n.1, p.215-225, Jan. 1989.

OLIVEIRA, M.; CURI, N.; FREIRE, J.C. Influência do cultivo na agregação de um Podzólico VermelhoAmarelo textura média/argilosa da região de Lavras (MG). Revista Brasileira de Ciência do Solo, Campinas, v.7, n.3, p.317-322, set./dez. 1983.
PAEZ, M.; ILDEFONSO, P.S. Evaluación de la eficiencia de índices de erodabilidad en suelos agrícolas en Venezuela. Agronomia Tropical, Maracay, v.42, n.1/2, p.27-40, 1992.

RENARD, K.G.; FOSTER, G.R.; YODER, D.C.; McCOOL, D.K. Rusle revised: status, question, answers and the future. Journal of Soil and Water Conservation, Ankeny, v.49, p.213-220, 1994.

RESENDE, M. Aplicações de conhecimentos pedológicos à conservação de solos. Informe Agropecuário, Belo Horizonte, v.11, n.128, p.3-18, ago. 1985.

RESENDE, M.; CURI, N.; REZENDE, S.B.; CORRÊA, G.F. Pedologia: base para distinção de ambientes. 2.ed. rev. e ampl. Viçosa: Núcleo de Estudo de Planejamento e Uso da Terra/Universidade Federal de Viçosa, 1997. 367p.

RÖMKENS, M.J.M.; ROTH, C.B.; NELSON, D.W Erodibility of selected clay subsoils in relation to physical and chemical properties. Soil Science Society of America. Journal, Madison, v.41, n.5, p.954-960, Sept./Oct. 1977.

ROTH, C.B.; NELSON, D.W.; RÖMKENS, M.J.M Prediction of subsoil erodibility using chemical, mineralogical and physical parameters. Washington, 1974. 111p.

SAS INSTITUTE. SAS language and procedures: Usage version 6. Cary, NC, 1985. 373p.

SCHWERTMANN, U. Differenzierung der Eisenoxide des Bodens durch Extraktion mit AmmoniumoxalatLösung. Zeitschrift für Pflanzenernährung Düngung Bodenkunde, Berlin, v.105, n.3, p.194202, 1964.

SHELDRICK, B.H.; McKEAGUE, J.A. A comparison of extractable $\mathrm{Fe}$ and $\mathrm{Al}$ data using methods followed in the USA and Canada. Canadian Journal of Soil Science, Ottawa, v.55, n.1, p.77-78, Feb. 1975.

SILVA, I.F.; ANDRADE, A.P.; CAMPOS FILHO, O.R. Erodibilidade de seis solos do semi-árido paraibano obtida com chuva simulada e método nomográfico. Revista Brasileira de Ciência do Solo, Campinas, v.10, n.3, p.283-287, set./dez. 1986.

SILVA, M.L.N. Erosividade da chuva e proposição de modelos para estimar a erodibilidade de latossolos brasileiros. Lavras: UFLA, 1997. 154p. Tese de Doutorado.

Pesq. agropec. bras., Brasília, v.34, n.12, p.2287-2298, dez. 1999 
SILVA, M.L.N.; CURI, N.; MARQUES, J.J.G.S.M.; LIMA, L.A.; FERREIRA, M.M.; LIMA, J.M. Resistência ao salpico provocado por impacto de gotas de chuva simulada em latossolos e sua relação com características químicas e mineralógicas. Ciência e Prática, Lavras, v.19, n.2, p.348-354, abr./jun. 1995.

SILVA, M.L.N.; CURI, N.; OLIVEIRA, M.S.; FERREIRA, M.M.; LOMBARDI NETO, F. Comparação de métodos direto e indiretos para determinação da erodibilidade em latossolos sob cerrado. Pesquisa Agropecuária Brasileira, Brasília, v.29, n.11, p.1751-1761, nov. 1994.

UEHARA, G.; GILLMAN, G. The mineralogy, chemistry and physics of tropical soils with variable charge clays. Boulder: Westview, 1981. 170p.

WEAVER, R.M.; SYERS, J.K.; JACKSON, M.L. Determination of silica in citrate-bicarbonatedithionite extracts of soils. Soil Science Society of
America. Proceedings, Madison, v.32, n.5, p.497501, Sept./Oct. 1968.

WISCHMEIER, W.H.; JOHNSON, C.B.; CROSS, B.V. A soil erodibility nomograph for farmland and construction sites. Journal of Soil and Water Conservation, Ankeny, v.26, n.5, p.189-193, Sept./ Oct. 1971.

WISCHMEIER, W.H.; MANNERING, J.V. Relation of soil properties to its erodibility. Soil Science Society of America. Proceedings, Madison, v.33, n.1, p.131-137, Jan./Feb. 1969.

WISCHMEIER, W.H.; SMITH, D.D. Predicting rainfall erosion losses: a guide to conservation planning. Washington: United States Department of Agriculture, 1978. 58p. (Agriculture Handbook, 537).

YOUNG, R.A.; MUTCHLER, C.K. Erodibility of some Minnesota soils. Journal of Soil and Water Conservation, Ankeny, v.32, n.4, p.180-182, July/ Aug. 1977. 